\section{Online links \\ Dicer}

http://www.ncbi.nlm.nih.gov/entrez/ query.fcgi?db=gene\&cmd=Retrieve \&dopt=Graphics\&list_uids=23405

env

http://www.ncbi.nlm.nih.gov/entrez/ query.fcgi? $\mathrm{db}=$ gene\&cmd=Retrieve \&dopt=Graphics\&list_uids $=155971$

Tat

http://www.ncbi.nlm.nih.gov/entrez/ query.fcgi? $\mathrm{db}=$ gene\&cmd=Retrieve \&dopt=Graphics\&list_uids $=155871$

HIV

\title{
Silencing HIV the natural way
}

A recent paper in Immunity describes the first natural example of RNA silencing in mammalian host-virus interactions. This evolutionarily ancient form of nucleic-acid-based immunity has previously been found in plants and invertebrates. But the demonstration that human cells can physiologically silence HIV-1 RNA highlights the universal importance of this system.

RNA silencing (also known as RNA interference, RNAi) in invertebrates is based on the cleavage of double-stranded RNA precursors by the RNase Dicer to form short interfering RNAs (siRNAs) of $\sim 21$ nucleotides; siRNAs then induce the degradation of complementary target RNAs, leading to post-transcriptional gene silencing. Silencing of viral genes is a natural antiviral response in plants and insects, using siRNA derived from the viral genome by the host processing machinery.

To examine the relevance of this response in immunity to HIV-1, the authors looked for sequences in the viral genome that could form the RNA-duplex precursors that are necessary for cleavage by host Dicer. They found one sequence in HIV-1, in the env gene, that formed a 19-base-pair duplex, and this sequence was functionally recognized by Dicer in vitro. Furthermore, the Dicer cleavage product - viral siRNA (vsiRNA) - was detected in HIV-infected T cells but not mockinfected cells.

To test whether the vsiRNA could mediate RNAi, they used constructs of its target RNA sequence fused to enhanced green fluorescent protein (EGFP), so that cleavage of the target RNA would prevent transcription of the downstream EGFP-encoding RNA. The addition of vsiRNA precursors led to a dose-dependent silencing of EGFP expression, and these precursors could also silence expression of the cognate target HIV-1 env mRNA.

As HIV-1 successfully replicates in human cells to high levels of cellfree RNA, it must be able to defend against this host-exploited RNAi. Synthetic siRNA precursors specific for the HIV-1 trans-activation response element (TAR) were ineffective at suppressing EGFP expression from a TAR-EGFP transcript when the HIV-1 transactivator (Tat) was included in the assay, and the suppressive function of Tat was also shown for other non-TAR sequences. This indicates that Tat is a general suppressor of RNA silencing (SRS). This was not due to the transcription of a downstream effector, as a point mutant of Tat without transcriptional activity could still act as an SRS. Instead, Tat was shown in vitro to target the host RNAi mechanism upstream of vsiRNA generation, at the level of Dicer.

Another Tat mutant was transcriptionally proficient but lacked SRS activity. Recombinant HIV containing this mutated form of Tat replicated less efficiently than HIV containing wild-type Tat. However, the difference was only slight, indicating that HIV infection is controlled by a range of host factors, one of which is the balance between host-exploited RNAi and viral SRS. When this balance was disrupted by the addition of oligonucleotides that inhibit vsiRNA into HIV-1-transfected cells, there was a dose-dependent increase in viral replication.

The authors suggest that the finding of only one duplex sequence in the HIV-1 genome indicates the strong selective pressure exerted by host RNAi against such sequences, and that the remaining sequence must have a crucial non-mutatable function for HIV-1, requiring the maintenance of a viral SRS.

Kirsty Minton, Associate Editor, Nature Reviews Immunology

\section{(2) References and links} ORIGINAL RESEARCH PAPER Bennasser, $Y$., Le, S.-Y., Benkirane, M. \& Jeang, K.-T. Evidence that HIV-1 encodes an siRNA and a suppressor of RNA silencing. Immunity 22, 607-619 (2005) 